Arxiu d'Etnografia de Catalunya, $n^{\circ} 7,1989,138-149$

ISSN: 0212-0372. EISSN: 2014-3885

http://antropologia.urv.cat/revistarxiu

ARXIU D' ETNOGRAFIA DE CATALUNYA $1989,7: 137-149$

\title{
ENVASES ALIMENTARIOS O ALIMENTOS ENVASADOS
}

\author{
Arachu Castro
}

Area de Antropologia Social

Universidad de Barcelona 
L' alimentation occidentale est passée directement "de la barbarie mediévale a la decadence industrielle" (Goody 1984, p. 251).

Los envases se han convertido en las ultimas décadas en un elemento imprescindible de la compra alimentaria. Hoy en dia es imposible, salvo en contadas ocasiones, adquirir alimentos per se. Se ha 1 legado a un punto en que también se tienen que comprar los envases. Esta situación no s6lo repercute en la economía doméstica, sino que significa un cambio profundo en el comportamiento alimentario de la población. La evolución de las técnicas de envasado, junto con las de conservación de alimentos, transforman los procesos de fabricación, distribución, compra y preparación de los alimentos, tanto a nivel empresarial como familiar.

Los estudios socio-culturales sobre la relación entre la evolución del envase y el comportamiento alimentario son recientes $^{1}$. En el caso de la población española no hay estudios socioculturales sobre esta relación, aunque hay datos estadisticos sobre tipos de productos alimentarios en función del establecimiento donde se efectúa la compra, publicados por el CIS (1988) y por la Dirección General de Politica Alimentaria (1989).

La primera parte del trabajo introduce el concepto de envase desde el punto de vista de la producción y la segunda parte desde la legislación española. El tercer apartado analiza, desde la perspectiva del comerciante, la evolución de la forma de entrega de los alimentos. La parte cuarta se dedica a la evolución del envase en el mercado español. Al final se hacen algunas reflexiones sobre cómo ha repercutido la evolución de los envases en la alimentación diaria de la población española.

\section{El envase y la producción de alimentos.}

Envasar los alimentos tiene el objeto principal de transportarlos, almacenarlos y conservarlos. Pero estas funciones se han ampliado en estas ultimas dos o tres décadas debido al enorme crecimiento del envasado, acompañado de avances en materiales y en maquinaria. No sólo es un recipiente, sino que "el envase debe proteger 10 que vende, y vender lo que protege" (Briston-Neill 1972). Hay una amplia interacción entre el envasado por un lado y la distribución de la venta al detall y al por mayor por el otro. Esto, junto con la creciente integración del envasado con el diseño del producto, la fabricación, el márketing y la distribución, significa que su

1 Ver entre otros Bender (1988), Bengtsson (1988), Gaull (1988), Goody (1984), Hesseltine (1988), Miller (1988), y Steinkraus (1988) 
desarrollo es vital para la dirección empresarial.

El envase de un producto, desde la perspectiva empresarial, se basa principalmente en cinco criterios. La apariencia es el aspecto del envase más importante desde el auge de los supermercados. Identifica el producto en la cadena de distribución y especialmente cuando llega al consumidor -ya sea por su transparencia $y / 0$ por su etiquetado. Informa sobre su composicion $y$ sobre las instrucciones de uso, según sean los requerimientos legales, $y$ sobre la marca $y$ el nombre del fabricante. Además, puede ayudar a promover la venta.

La protección que ofrece varía en función del tipo de producto y del sistema de distribución. El envase actúa como protector del producto contra influencias perjudiciales como son: captación de agua, absorción de vapor de agua, desecación, penetración de aire, polvo y microorganismos, contaminación por insectos $y$ por animales domésticos, $y$ acción de la luz. La funcionalidad del envase facilita el uso del producto, desde abrirlo hasta, en algunos casos, cocinarlo. El coste debe incluir el del transporte y el del almacenamiento de los envases vacios, el de su reutilización -asi como el control de calidad de los envases devueltos para ello- y el de las pérdidas debidas a la rotura del envase. La finalidad del envase es que el producto llegue al consumidor en buen estado y al minimo coste.

La desechabilidad del envase es el tema mas polémico de todos. El uso de envases desechables contribuye a aumentar la polución ambiental y representa un gasto importante de los recursos del planeta. Algunos de los factores que contribuyen a aumentar la cantidad de basura formada por envases vacios son la existencia de productos hiper-envueltos y la proliferación de envases sin retorno. En el caso de los primeros, el material de envasado podría reducirse haciendo un cambio de diseño, con lo que se gastarian menos recursos y se reduciria el coste de dicho envasado. El caso de los envases sin retorno es más delicado ya que alla fabricante les resultan más económicos que los retornables. Estos últimos no siempre se devuelven --bien porque los consumidoresas les dan otra utilidad, porque se rompen o porque los almacenan en casa-- suelen ser más pesados y más costosos que los no retornables, y además presentan unos gastos de acondicionamiento. Los fabricantes consideran que los envases sin retorno tienen cada vez una mayor aceptación por parte de los compradores, ya que son más higiénicos y más cómodos porque son de un-solo-viaje. Aunque no hay ningún estudio socio-cultural que analice el consumo de alimentos en función de que estén contenidos en envases retornables 0 en no retornables, la tendencia actual indica que la fabricación $y$ el consumo de envases alimentarios no retornables va en aumento, especialmente porque favorecen la economia de la industria alimentaria, aunque hay que tener en cuenta que su proliferacion presenta el inconveniente de que aumenta el volumen de los vertidos y que las soluciones dadas hasta la fecha son insuficientes ${ }^{2}$.

2 Reciclar el material de envasado suele ser bastante práctico en el caso del papel, el aluminio y el cristal; no asi en el caso del plástico, que presenta costes de reciclaje elevados. Darles una aplicación diferente, como es mezclar el 


\section{El envase y la legislación alimentaria}

El objeto principal de las leyes sobre alimentos es conseguir que todos los productos que se ofrecen al consumidora sean seguros, no adulterados y que vayan correctamente etiquetados. El Estado dicta las prescripciones legales que garanticen los derechos del consumidor y aseguren, con las pertinentes medidas de autoridad, el cumplimiento de estas prescripciones. En España, la normativa legal está constituida por el CAE (Código Alimentario Español) y por la Ley General para la Defensa de los Consumidores $y$ Usuarios (BOE del 19 de julio de 1984, Ley 26/1984). Además, la Generalitat de Catalunya ha publicado una Ley sobre la higiene y el control alimentario (DOGC del 14 de julio de 1983 , Ley $15 / 1983$ ).

El CAE, creado en septiembre de 1967, es:

"el cuerpo orgánico de normas básicas y sistematizadas relativas a los alimentos, condimentos, estimulantes y bebidas, sus primeras materias correspondientes $y$, por extension, a los productos, materias, utensilios y enseres de uso y consumo doméstico" (Código Alimentario 1988: 17).

Pero, en realidad, el Estado Español crea el código Alimentario en 1955. Es el más avanzado de Europa, pero se realiza sin contar con la colaboración de quienes verdaderamente manejan los productos alimenticios. Ni fabricas, ni comercios, ni hoteles, ni restaurantes, ni bares están en condiciones dé cumplir sus exigencias, ya que la falta de recursos econ6micos e higiénicos es considerable en esa etapa de reconstruccion economica.

Como resultado del fracaso que supone el intento de poner en marcha el código, tienen que rehacerse las Reglamentaciones Sanitarias de cada industria, comercio $y$ otros servicios, pero esta vez se cuenta con el asesoramiento de fabricantes, comerciantes y hoteleros agrupados en distintas comisiones. Se dan plazos para que se ajusten a las exigencias del código, y el $C A E$ no se pone verdaderamente en marcha hasta transcurridos doce afios (San Juan 1989).

En los años setenta, y contando también con las comisiones de fabricantes, se toman otras tres medidas de importancia: la obligatoriedad de colocar en los envases las fechas de caducidad, la prohibición de la venta a granel y la imposición del carnet de Manipulador de Alimentos para todo el personal del sector fabricante o comerciante de alimentación.

cristal de los envases con asfalto o el plastico con cemento, es una manera de esconder los envases. Deshacerse del material de envasado es la forma que tiene más futuro para la industria. Puede realizarse de tres maneras: hundiendo el material en el mar, enterrándolo o incinerándolo, con todos los problemas ecologicos que ello supone. Hacer envases biodegradables, a pesar de sus ventajas, presenta el inconveniente de que cada material necesita un tiempo diferente para desintegrarse. La proliferacion de envases alimentarios es una más de las consecuencias de la decadencia industrial. 
Sin embargo, el control de estas normas por parte del Gobierno no es muy estricto, ya que sigue existiendo la venta incontrolada de productos alimenticios por calles, pisos $y$ mercadillos. Bajo el nombre de "natural" y "casero" se venden, entre otros productos: pan, pastas, embutidos, jamones, quesos $y$ aceites. Esta forma de economia sumergida la critican los fabricantes y comerciantes del sector alimentario, quienes la tildan de ilegal e intrusista. Tanto los fabricantes como los comerciantes no ganan la batalla hasta la aparición del síndrome tóxico, que en un período de ocho años produce más de 600 muertes y unos 25.000 afectados con lesiones. En 1990 todavia no se conoce el agente causal, pero oficialmente se considera que el vehiculo es el aceite de colza desnaturalizado.

con el sindrome toxico, las fechas de caducidad cobran importancia a todos los niveles. El Gobierno toma medidas más severas, no s6́lo con respecto al etiquetado, sino también con respecto a la venta ambulante y a granel -lo cual beneficia a los comerciantes que ya cumplian las normas sanitarias. Estos cambios pueden suceder gracias a que la población toma conciencia de la importancia de las fechas de caducidad: el sindrome tóxico sensibiliza a la población española de la importancia de adquirir alimentos en los establecimientos autorizados. La aureola de "naturalidad" y "tradición" de la venta ambulante, en especial la del aceite, se empieza a desvanecer, aunque no desaparece por completo: en 1988 un $2.77 \%$ de los hogares españoles compran alimentos en mercadillos, venta callejera y venta a domicilio (Dirección General de Politica Alimentaria 1989, p. 54).

Los conceptos de naturalidad y tradición se incorporan al marco de la legalidad: la población empieza a consumir productos de estilo casero preparados por la industria alimentaria. La tendencia actual en las preferencias alimentarias es la de los productos "sin" y "con", en los que convergen tanto los productos que buscan la naturalidad - sin aditivos, sin colorantes, sin conservantes, con fibra- como los productos alimenticios altamente manipulados en los que se llegan a sustituir, eliminar - incrementar los componentes de base: salsas mayonesas sin huevo, bebidas refrescantes sin azúcar y sin calorias, leche descremada sin colesterol, $y$ cereales $y$ margarinas con suplementos vitaminicos.

La oferta de estos productos en envases de distintos tamaños multiplica la variedad de alimentos disponibles, que se van integrando en las dietas tradicionales. Faltan estudios sobre los sectores de población en que estos nuevos productos tienen una mayor aceptación, pero datos de otros paises indican que la población recién emigrada a un núcleo urbano los incorpora de forma rapida en su alimentación (Garine 1975, McElroy-Townsend 1985, Castro 1989b). La explicación de estos estudios es que las pautas alimentarias de la poblacion emigrante se desestructuran por la ausencia de productos del lugar de origen y por la falta de integración social de esos grupos. En esa etapa de transición entre su alimentación tradicional y la del lugar de acogida es fácil que, por la falta de equilibrio de sus pautas de alimentación, los emigrantes incorporen nuevos hábitos de consumo alimentario. 


\section{Entrega y conservación de alimentos.}

La historia del envase se remonta logicamente a tiempos antiguos: hojas, pieles de animales, cestas, telas, madera, papel y cristal; el uso de este último se remonta a la época de los comerciantes fenicios (Briston-Neill 1972). El metal entra en escena más tarde: se empieza a utilizar en Francia a principios del siglo XIX (Goody 1984). Y el plástico-junto con el envasado al vacio- es la contribución del presente siglo a las técnicas de envasado.

Hasta la Guerra Civil española, la conservación y envasado de alimentos no cambia de manera significativa con respecto a siglos anteriores. Los alimentos perecederos crean problemas de conservación, pues no se cuenta con medios suficientes -s6lo con barras de hielo- para luchar contra la putrefacción. A excepción de los productos alimenticios conservados con técnicas como la salazón, el ahumado, la deshidratación, o las conservas en aceite o en vinagretas, hay que vender y consumir rápidamente tanto la carne como el pescado $y$, en menor medida, la fruta y la verdura.

Los animales se sacrifican en el Matadero Municipal por la tarde, y el veterinarioa es quien determina si son aptos para el consumo o no. Se aprovecha la baja temperatura de la noche para que la carne se oree $y$ al día siguiente se pone a la venta $y$ se procura vender en el día 0 , a lo sumo, a la mañana siguiente, ya que de no ser asi rápidamente comienza el proceso de putrefacción y su pérdida. El pescado llega a los establecimientos recién capturado en cajas destapadas de madera llenas de hielo picado que lo cubre. Se despacha rápidamente: lo que no se puede vender en el día es de dificil venta porque en seguida comienza a estropearse y a desprender mal olor. Tanto el pescado como la carne se entregan al consumidor envuelto en una hoja de árbol fresca, en papel de estraza o en papel de peribdico. Las frutas y las verduras no presentan gran problema porque se consumen frescas. También se emplean para hacer conservas caseras.

Los alimentos no perecederos son las legumbres y los frutos secos, que se consideran de duración eterna. Estos dejan de venderse cuando, al cabo del tiempo, les atacan ciertos parásitos. Por otro lado hace ya varios años que fur.cionan industrias conserveras. Al igual que con las conservas caseras, se supone que su duración es ilimitada, aunque cabe la posibilidad de que a veces se estropeen.

Después de la Guerra Civil llegan a España las primeras neveras. Son unos armarios forrados de corcho aislante en los que se colocan los alimentos perecederos y que, con hielo en su interior $y$ un pequeño depósito para recoger el agua del deshielo, se logra hacer durar durante un par de días más el buen estado de esos alimentos. También se utilizan las fresqueras, que son unos armarios construidos de forma saliente en una pared exterior de las viviendas $y$ que se abren desde el interior. Están orientados al norte, $y$ mantienen frescos los alimentos.

A partir de los años cuarenta, más que hablar de evolución habria que hablar de revolución en el terreno de la conservación y envasado de alimentos (San Juan 1989). La revolución de la conservación en frio llega a la industria $y$ a las unidades domésticas. Comienzan a venderse los primeros frigorificos familiares, caros e imperfectos, pero que paulatinamente se 
abaratan hasta ponerse al alcance de casi todo el mundo.

En los años setenta se empiezan a comercializar en España las primeras neveras familiares con capacidad de congelar los alimentos -las de tres y cuatro estrellas. Esto supone en la década de los ochenta una transformación importante en el comportamiento alimentario de un sector de la población, especialmente cuando aparecen los congeladores separados del resto del frigorifico, o en un mismo mueble pero con dos motores diferentes. Los datos más recientes sobre equipamiento del hogar indican que un $95 \%$ de los hogares españoles disponen de nevera (CIS 1988:61), aunque no especifican de qué tipo es. Estudios antropológicos indican que mientras una parte de la población siente rechazo a congelar los alimentos porque lo consideran un elemento destructor (Carrasco 1990), la congelación se impone como una faceta indispensable de la vida urbana de otro sector. Congelar alimentos significa realizar compras con menor frecuencia, tener una mayor disponibilidad de alimentos en casa, y preparar ciertos alimentos en menor tiempo-como es el caso de las verduras congeladas. Por todas estas razones, la congelación la aceptan las personas a las que su previsión en la compra de productos congelados les permite una mayor improvisación a la hora de cocinar, o bien simplemente a las que no les gusta ir a comprar. Estos factores se acentúan con la utilización de los hornos microondas. Pero mientras a algunas personas les guste salir a comprar, sobre todo diariamente, la congelación no puede extenderse a toda la población.

\section{Historia del envase.}

La breve historia de la entrega y conservación de alimentos sugiere que la primera función que tiene el envase es la de permitir que el comerciante adquiera el producto -si no lo fabrica él mismo-- y que lo entregue a su cliente. Se trata de envolver los alimentos para evitar tocarlos directamente hasta llegar a casa ${ }^{3}$. Esto va acompañado por el hecho de que el cliente se deja asesorar por el tendero: los productos de venta no están siempre a la vista, y el tendero sirve al cliente un producto determinado en función de la calidad o precio que éste le pida. El trato es diario y personal, ya que por lo general la gente compra siempre en el mismo colmado o tienda de ultramarinos, que suele ser el que está más cerca de casa.

Los envases alimentarios empiezan a transformarse cuando mejoran las vías de comunicación y las técnicas de conservación de alimentos. Estos deben estar preparados para poder ser transportados a mayores distancias que las habituales, con 10 cual, transporte y envases encarecen el producto al mismo tiempo, a pesar de que en esta época de reconstrucción económica para la vida española no se puede permitir el desprenderse de los envases sin reutilizarlos. No sólo cambian los envases, que deben ser más adecuados, sino que empiezan a utilizarse todo tipo de aditivos que permitan conservar los alimentos durante más tiempo. A estos factores hay que añadir el hecho de que esto supone un aumento

3 Hoy en día la forma de entrega del pan de panadería es la que más se asemeja a esa modalidad. 
de la variedad de alimentos disponibles, tanto a nivel geográfico como estacional.

A principios de los años sesenta, un nuevo tipo de establecimientos empieza a surgir en las grandes ciudades españolas: el autoservicio y el supermercado --éste último se diferencia por su mayor volúmen de-ventas. En esta primera época no tienen aceptación. La razón más aceptada para este fenómeno es que el trato de estos nuevos establecimentos es despersonalizado, pero no se disponen de estudios realizados desde el punto de vista del consumidora española. Todos los productos alimenticios están a la vista del clientea, y éstea debe decidir la compra sin el consejo del tenderoa. Conscientes de ello, los fabricantes empiezan a utilizar envases que llamen la atención del clientea. Hacen mayores descuentos a los comerciantes si sitúan sus productos en lugares estratégicos del autoservicio o supermercado. Es aqui cuando la presentación del alimento cobra importancia, cuando empieza la función publicitaria del envase.

Esta función va a más cuando se supera el fracaso inicial de este tipo de establecimientos, que no sucede en los núcleos urbanos hasta principios de los años setenta. En esa época confluyen dos fenómenos importantes. En primer lugar, la entrada en una proporción ya apreciable de la mujer en el mundo laboral: cuando ésta empieza a trabajar fuera de casa, deja de disponer del tiempo que tenía anteriormente para hacer la compra diaria. A un sector de mujeres -que hipotéticamente coincide con las partidarias de la congelación- les resulta más práctico comprar menos veces a la semana, y en un establecimiento donde se pueda comprar de forma más rápida -es decir, evitando las largas $y$ controladoras conversaciones con los tenderos- y más económica. En segundo lugar, la aparición en el mercado de las primeras neveras familiares: las neveras permiten conservar durante más días los alimentos 0 , 10 que es 10 mismo, permiten que las compras de alimentos sean de mayores cantidades.

Los supermercados son aceptados por las personas que ya se relacionan socialmente en su actividad laboral, y para quienes comprar no es más que un trámite. Por el contrario, hay personas para quienes la compra de alimentos es un buen momento pa a las relaciones sociales -posiblemente porque no las tienen en su actividad laboral- $y$ porque comprar les permite "reencontrar el mundo femenino", ausente en según qué tipo de trabajo (Carrasco 1990). Son personas que, aunque trabajan una jornada completa fuera de casa, están más rato para comprar del estrictamente necesario. Hay que tener en cuenta que datos recientes de una muestra de Barcelona indican que en el $92.7 \%$ de las familias la mujer realiza la compra alimentaria: en el 80.8 \% de los casos compra sólo la mujer, en el 13.4 \% compran tanto la mujer como el varón, y en el resto compra sólo el varón (Castro 1989a). La compra alimentaria es una tarea eminentemente femenina, y si perdura asi a pesar del doble trabajo de muchas mujeres españolas -en y fuera de casa-- es probablemente porque la mujer encuentra algún tipo de bienestar al comprar la comida-, serla demasiado simplista afirmar que si no 10 hace ella no lo hace nadie.

La competencia entre fabricantes del mismo ramo ya no es únicamente por la calidad del producto, sino por presentarlo en un envase que lo venda mejor. Aqui confluyen diversos factores: primero, la importante función publicitaria a la que se ha hecho 
referencia. Segundo, la evolución de los envases permite presentar los alimentos en paquetes de distintos formatos y que los fabricantes empiecen a jugar con el factor tamaño-peso: la apariencia del envase ya no es sólo por su colorido y por sus letras, sino por su tamaño, que puede dar la sensación de mayor peso que el que tiene en realidad. Desde los envases de tamaño familiar, que son en proporción los más económicos, hasta los de tamaño individual, que son los más caros, cualquiera puede encontrar en la actualidad española el envase que mejor se adapte al tamaño y a la forma de consumo de su unidad doméstica. Tercero, la introducción de los combinados de cartón y plástico sustituye a las antiguas latas retornables -como las de las galletas- que además de incorporar una mayor higiene, mejora la conservación de los alimentos.

Cuarto, así empieza la era de los envases desechables, la era de la comodidad. La gente prefiere pagar un poco más y no tener que almacenar y cargar de vuelta al supermercado con los envases vacios. Es el auge de las botellas de plástico y de los envases tetra-brik, que paulatinamente sustituyen a las pesadas botellas de cristal. Tan importante es la comodidad, que a primera vista sorprende que se llegue a vender vino en envases de cartón, a pesar del arraigo que tienen las botellas de vino en la sociedad española. Una explicación es que los productos básicos de un sistema de alimentación determinado no actúan como marcadores sociales. Comprar una u otra marca comercial de harina, azúcar, huevos o sal no interviene en la presentación social del comprador. Por este motivo, las personas para quienes el vino no es un marcador social pueden estar más predispuestas a comprar vino en envases de cartón. Suelen ser personas en proceso de aculturación, como es el caso de los emigrantes (Carrasco 1982, basado en Nikod-Douglas 1974).

\section{¿Qué ocurre con los envases?}

Los envases cambian porque, en primer lugar, los fabricantes ven que son un arma importante para comercializar sus productos. Esto es posible gracias a un cambio en las técnicas de envasado, que incorporan nuevos materiales, a la progresiva implantación de establecimientos alimentarios en los que el propio clientea se sirve los productos que quiere, $y$ a la aceptación de estos cambios por los compradores y los consumidores. El primer punto es imprescindible para que se inventen los envases alimentarios, el segundo los lanza a la fama y el tercero los compra y los consume.

De la importancia del primero se ha hablado ya, y de la del segundo se constata mejor si se compara con otra industria, la farmacéutica. En ese sector también se incorporan las nuevas técnicas $y$ materiales de envasado, pero no se da el mismo proceso. Aquí se refiere al caso español, en el que la venta de medicamentos pasa necesariamente por el mostrador. Los productos farmacéuticos no varían mucho su apariencia externa, ya que son productos de mostrador, en el que el farmacéutico hace de intermediario entre la prescripción médica y el comprador. Éstea no elige el medicamento que quiere, sino que la compra está decidida previamente por el médico. Los fabricantes de productos farmacéuticos no tienen la necesidad de crear unos envases que 
vendan el producto -habría que hacer una excepción con los de dermofarmacia $y$, en general, con los OTC, que son los que no cubre la Seguridad Social- sino que aplican las nuevas técnicas a una función primordial del envasado, que es la protección y conservación del producto.

El tercer punto representa una transformación del comportamiento alimentario que se ha explicado, en una primera aproximación, por la incorporación masiva de la mujer al mundo laboral y por 10 que a un sector de la población femenina le favorece la nueva forma de disponibilidad de alimentos.

\section{Establecimientos utilizados en primer lugar para la compra de alimentos y bebidas, según edad. Basado en CIS $(1988: 40)$.}
18 a 25
26 a 40
41 a 60
más de 60

\begin{abstract}
Mercados y
ultramarinos

Supermercados e

hipermercados
\end{abstract}

Otros

TOTAL
5

100
50

43

7

100
66

30

4

100

Los supermercados e hipermercados son el tipo de establecimiento más frecuentado, y de forma mayoritaria, por las mujeres más jovenes: entre el 51\% y el 52\%. El resto de población acude principalmente a los establecimientos de tipo tradicional: la mitad de las mujeres entre 41 y 60 años, y la tercera parte de las mayores de 60. Con el paso de los años es de suponer que la frecuencia de compra en supermercados e hipermercados sea mayor, con lo cual el envase alimentario, causante de tan profundos cambios -culturales y ecologicos-continuará proliferando, y en una situación tal es dificil discernir si la tendencia de compra alimentaria está dirigida a los envases alimentarios o a los alimentos envasados.

\section{BIBLIOGRAFIA}

Bender, Arnold E.

1988 "The influence of food processing on nutrition".

En:Moyal, M.F.(ed) Alimentation et societés: Nouvelles Technologies. Proceedings of the Xth International

Congress of Dietetics. Paris: John Libbey, pp 243-250.

Bengtsson, Nils

1988 "Perspectives in large scale food preparation: The impact of food processing fundamentals and equipment development on food quality".

En Moyal, M.F.(ed) op. cit., pp 279-297.

Boletín oficial del Estado

1988 Código alimentario español. 
Madrid, BOE, $473 \mathrm{pp}$.

Briston, John H.- Neill, Terence J.

1972 Packaging management.

Essex, GB: Gower Press.

Carrasco i Pons, Silvia

1982 Una aproximación a la antropología de la alimentación: higienismo y alimentación obrera en Sabadell durante el cambio de siglo.

Tesis de Licenciatura.

Bellaterra: Universitat Autònoma de Barcelona, $234 \mathrm{pp}$.

1989 Comunicación personal.

Castro, Arachu S.

1989a "Estudio sobre los hábitos alimentarios y su relación con el nivel económico y el origen cultural en la población de Barcelona"

Ponencia presentada en el III Congreso de Sociología, San Sebastián, $17 \mathrm{pp}$.

$1989 \mathrm{~b}$ "A study on the issues that deal with the setting up of food choices: An experience with Mexican-American farmworkers that live in Tulare County, California", Informe presentado a la CIRIT, de la Generalitat de Catalunya, $13 \mathrm{pp}$.

CIS

1988 "Actitudes y comportamientos de los españoles ante el consumo"

Estudios $y$ encuestas $14,170 \mathrm{pp}$.

Dirección General de Política Alimentaria

1989 Consumo alimentario en España 1988.

Madrid: Ministerio de Agricultura, Pesca y Alimentación, Tomos I y II,

1644 pp.

ESADE

1989 El sector distributivo alimentario en España y Europa. Mimeo, $52 \mathrm{pp}$.

Finkelstein, Joanne

1989 Dining out.

Cambridge, GB: Polity Press, 199 pp.

Garine, Igor de

1975 "Changing food habits?".

Nutrition, Proceedings of the 9 th International Congress of Nutrition. Mexico 1972.

Basilea: Karger, Vol. 4, pp 239-247.

Gaull, G.

1988 "Foods for the future: Role of industry" En Moyal, M.F.(ed) op. cit., pp 333-334. 
Generalitat de Catalunya

1988 Habits alimentaris $i$ consum d'aliments a Catalunya.Llibre blanc.

Barcelona: Servei de Promoció de la Salut,

Goody, Jack

1984 Cuisines, cuisine et classes.

Paris: Centre Georges Pompidou.

Hesseltine, C.W.

1988 "Transfer of food fermentation technology".

En Moyal, M.F. (ed) op. cit., pp 159-165.

Kotler, Philip

1986 Dirección de mercadotecnia: Análisis, planeación y control. México: Diana, 867 pp.

McElroy, Ann y Townsend, Patricia K.

1985 Medical anthropology in ecological perspective.

Boulder, Colorado: Westview, $482 \mathrm{pp}$.

Miller, Sanford A.

1988 "New foods: Old problems".

En Moyal, M.F.(ed) op. cit., pp 223-232.

Nikod, Michael - Douglas, Mary

1974 "Taking the biscuit: The structure of British meals" New Societies 19.

San Juan de Maeztu, Arturo

1989 Evolución de la conservación y envasado de alimentos. Mimeo, $12 \mathrm{pp}$.

Steinkraus, Keith H.

1988 "Contribution of indigenous fermented foods to the world dietary".

En: Moyal, M.F. (ed) op. cit.,pp.167-198. 